\title{
The Gaia-ESO Survey: the first abundance determination of the pre-main-sequence cluster gamma Velorum ${ }^{\star} \star \star \star$
}

L. Spina ${ }^{1,2}$, S. Randich ${ }^{1}$, F. Palla ${ }^{1}$, G. G. Sacco $^{1}$, L. Magrini ${ }^{1}$, E. Franciosini ${ }^{1}$, L. Morbidelli ${ }^{1}$, L. Prisinzano ${ }^{3}$, E. J. Alfaro ${ }^{4}$, K. Biazzo ${ }^{5}$, A. Frasca ${ }^{5}$, J. I. González Hernández ${ }^{6,7}$, S. G. Sousa ${ }^{8,9}$, V. Adibekyan ${ }^{8}$, E. Delgado-Mena ${ }^{8}$, D. Montes ${ }^{10}$, H. Tabernero ${ }^{10}$, A. Klutsch ${ }^{5}$, G. Gilmore ${ }^{11}$, S. Feltzing ${ }^{12}$, R. D. Jeffries ${ }^{13}$, G. Micela ${ }^{3}$, A. Vallenari ${ }^{14}$, T. Bensby ${ }^{11}$, A. Bragaglia ${ }^{15}$, E. Flaccomio ${ }^{13}$, S. Koposov ${ }^{10}$, A. C. Lanzafame ${ }^{16}$, E. Pancino ${ }^{15,17}$, A. Recio-Blanco ${ }^{18}$, R. Smiljanic ${ }^{19,20}$, M. T. Costado ${ }^{4}$, F. Damiani ${ }^{3}$, V. Hill ${ }^{18}$, A. Hourihane ${ }^{10}$, P. Jofré ${ }^{10}$, P. de Laverny ${ }^{18}$, T. Masseron ${ }^{11}$, and C. Worley ${ }^{11}$

1 INAF - Osservatorio Astrofisico di Arcetri, Largo E. Fermi, 5, 50125 Firenze, Italy e-mail: lspina@arcetri.astro.it

${ }^{2}$ Università degli Studi di Firenze, Dipartimento di Fisica e Astrofisica, Sezione di Astronomia, Largo E. Fermi, 2, 50125 Firenze, Italy

3 INAF - Osservatorio Astronomico di Palermo, Piazza del Parlamento 1, 90134 Palermo, Italy

${ }^{4}$ Instituto de Astrofísica de Andalucía-CSIC, Apdo. 3004, 18080 Granada, Spain

5 INAF - Osservatorio Astrofisico di Catania, via S. Sofia, 78, 95123 Catania, Italy

${ }^{6}$ Instituto de Astrofisica de Canarias (IAC), 38205 La Laguna, Tenerife, Spain

Depto. Astrofisica, Universidad de La Laguna (ULL), 38206 La Laguna, Tenerife, Spain

8 Centro de Astrofisica, Universidade do Porto, Rua das Estrelas, 4150-762 Porto, Portugal

9 Departamento de Física e Astronomia, Faculdade de Ciências, Universidade do Porto, Rua do Campo Alegre, 4169-007 Porto, Portugal

10 Departamento de Astrofisica, Universidad Complutense de Madrid (UCM), 28040 Madrid, Spain

11 Institute of Astronomy, University of Cambridge, Madingley Road, Cambridge CB3 OHA, UK

12 Lund Observatory, Department of Astronomy and Theoretical Physics, Box 43, 22100 Lund, Sweden

13 Astrophysics Group, Research Institute for the Environment, Physical Sciences and Applied Mathematics, Keele University, Keele, Staffordshire ST5 5BG, UK

14 INAF - Padova Observatory, Vicolo dell'Osservatorio 5, 35122 Padova, Italy

15 INAF - Osservatorio Astronomico di Bologna, via Ranzani 1, 40127 Bologna, Italy

16 Dipartimento di Fisica e Astronomia, Sezione Astrofisica, Universitá di Catania, via S. Sofia 78, 95123 Catania, Italy

17 ASI Science Data Center, via del Politecnico SNC, 00133 Roma, Italy

18 Université de Nice Sophia Antipolis, CNRS, Observatoire de la Côte d'Azur, BP 4229, 06304 Nice Cedex 4, France

19 Department for Astrophysics, Nicolaus Copernicus Astronomical Center, ul. Rabiańska 8, 87-100 Toruń, Poland

${ }^{20}$ European Southern Observatory, Karl-Schwarzschild-Str. 2, 85748 Garching bei München, Germany

Received 28 January 2014 / Accepted 21 April 2014

\section{ABSTRACT}

Context. Knowledge of the abundance distribution of star forming regions and young clusters is critical to investigate a variety of issues, from triggered star formation and chemical enrichment by nearby supernova explosions to the ability to form planetary systems. In spite of this, detailed abundance studies are currently available for relatively few regions.

Aims. In this context, we present the analysis of the metallicity of the gamma Velorum cluster, based on the products distributed in the first internal release of the Gaia-ESO Survey.

Methods. The gamma Velorum candidate members have been observed with FLAMES, using both UVES and Giraffe, depending on the target brightness and spectral type. In order to derive a solid metallicity determination for the cluster, membership of the observed stars must be first assessed. To this aim, we use several membership criteria including radial velocities, surface gravity estimates, and the detection of the photospheric lithium line.

Results. Out of the 80 targets observed with UVES, we identify 14 high-probability members. We find that the metallicity of the cluster is slightly subsolar, with a mean $[\mathrm{Fe} / \mathrm{H}]=-0.057 \pm 0.018$ dex. Although J08095427-4721419 is one of the high-probability members, its metallicity is significantly larger than the cluster average. We speculate about its origin as the result of recent accretion episodes of rocky bodies of $\sim 60 M_{\oplus}$ hydrogen-depleted material from the circumstellar disk.

Key words. stars: abundances - stars: pre-main sequence - open clusters and associations: individual: gamma Velorum techniques: spectroscopic

\footnotetext{
* Based on observations collected at the ESO telescopes under programme 188.B3002, the Gaia-ESO large public spectroscopic survey.

$\star \star$ Full Tables 1-4 are only available at the CDS via anonymous ftp to cdsarc.u-strasbg.fr (130.79.128.5) or via http://cdsarc.u-strasbg.fr/viz-bin/qcat?]/A+A/567/A55
}

\section{Introduction}

Open clusters are excellent tracers of the chemical pattern of the Galactic thin disk and its evolution (e.g., Friel 1995). The youngest clusters, the so-called pre-main-sequence (PMS) 
clusters with ages $\$ 50 \mathrm{Myr}$, are of particular interest since they are still close to their birthplaces and contain a homogeneous stellar population that has not had time to disperse through the Galactic disk. Thus, they are key objects to trace the current chemical composition of the solar neighborhood and its evolution in space and time.

Furthermore, and more specifically, determination of the chemical content of young clusters and star forming regions (SFRs) is critical for a variety of reasons that we summarize below. First, as originally discussed in the series of papers by Cunha and collaborators, knowledge of the abundance pattern allows us to investigate the common origin of different subgroups in a given association and it sheds light on the possible presence of enrichment caused by the explosion of a nearby supernova (Cunha et al. 1998; Biazzo et al. 2011a, and references therein). Indeed, in the triggered star formation scenario, newly formed massive stars belonging to a first generation of stars in a giant molecular cloud and ending their lifetime with supernova (SN) explosions, disperse the parent molecular cloud, preventing further star formation to occur in the immediate surroundings. At the same time, however, winds and $\mathrm{SN}$-driven shock waves are thought to trigger new star formation events at larger distances; since supernovae are major nucleosynthesis sites, these explosions, may also chemically enrich parts of the surrounding interstellar gas, and hence the newly formed second generation of stars (Cunha \& Lambert 1992, 1994, and references therein). Finding direct evidence of such selective enrichment in young clusters and SFRs would clearly give insights into a process that has occurred innumerable times in the past, not just in our own galaxy.

In addition, as in the case of old populations, the metal content of PMS clusters is a critical parameter for the determination of their distance, age, and individual stellar masses of their members. Metallicity has an effect on the internal stellar structure and on the surface properties through opacity: even relatively minor changes in the metal content could imply that there are differences in the derived cluster ages, distances, and masses (Sherry et al. 2008). These parameters in turn are critical for the determination of the initial mass function (IMF) and the star formation history within each region, as well as for investigating different properties such as disk lifetimes and the rotational evolution of young stars.

Third, recent theoretical studies have suggested that metallicity has an important impact on the evolution of circumstellar disks and their ability to form planets. For example, Ercolano \& Clarke (2010) have shown that disks should dissipate quickly in a metal-poor environment. Observational studies on the disk lifetime at low-metallicity are controversial. On the one hand, support for the theoretical predictions has been provided by Yasui et al. (2010) who found that the disk fraction $\left(f_{\mathrm{d}}\right)$ in low-metallicity clusters (with $[\mathrm{O} / \mathrm{H}] \sim-0.7$ ) declines rapidly and approaches $f_{\mathrm{d}} \sim 10 \%$ in $\lesssim 1 \mathrm{Myr}$, significantly earlier than solar-metallicity clusters for which the timescale is $\sim 5-7 \mathrm{Myr}$ (Sicilia-Aguilar et al. 2006; Mordasini et al. 2012). On the other hand, based on Hubble Space Telescope mass accretion rate measurements, Spezzi et al. (2012) suggest that disks in metalpoor clusters of the Large Magellanic Cloud may be long lived with respect to the Milky Way.

Finally, it is worth mentioning the correlation between metallicity and elemental abundances and the presence of giant planets around old solar-type stars (Gonzalez 1998; Santos et al. 2004; Johnson et al. 2010). In particular, Gilli et al. (2006), Neves et al. (2009), Kang et al. (2011) and Adibekyan et al. (2012a,b) have shown that the chemical differences between stars with and without exoplanets are not limited to the iron content, but also to the abundance of some refractory elements (e.g., $\mathrm{Mg}, \mathrm{Al}, \mathrm{Sc}, \mathrm{Ti}, \mathrm{V}$ and $\mathrm{Co}$ ). Thus, studying the metal content of nearby young clusters, hosting a number of T-Tauri stars with circumstellar disks and likely on the verge of forming planets, may provide useful constraints to studies of planet formation scenarios and their timescales.

In spite of all these exciting aspects, relatively few studies have addressed the issue of the metal content of PMS clusters and SFRs (see, e.g., James et al. 2006; Santos et al. 2008; D’Orazi et al. 2009; Biazzo et al. 2011a,b), rather mostly focusing on well studied, nearby regions like Orion and TaurusAuriga. A metallicity close to or slightly lower than the solar value has been measured for all these regions; interestingly, and at variance with older clusters, none of them appears to be metal-rich (Biazzo et al. 2011a). Since, as mentioned, only relatively few young clusters and only very few stars per region have high resolution abundance measurements, additional studies are clearly warranted.

The Gaia-ESO Survey (Gilmore et al. 2012; Randich \& Gilmore 2013) is a large public spectroscopic survey observing all the components of the Galaxy (bulge, thin and thick disks, and halo). The project makes use of the FLAMES spectrograph mounted at the VLT to obtain spectra of about $10^{5}$ stars, including candidate members of 90-100 open clusters. This large sample of observations will allow us to accurately study of the kinematical and chemical abundance distributions in the Milky Way and also to fully sample the age-metallicity-mass/densityGalactocentric distance parameter space within the open clusters selected. In this framework, the Gaia-ESO Survey represents a unique opportunity not only to extend the sample of young clusters and SFRs with metallicity and abundance determinations, but also to perform a homogeneous study based on a large stellar sample within each region. The Gaia-ESO Survey will provide a comprehensive and homogeneous view on the chemical contents of the youngest clusters in the Galaxy, based on the analysis of a large sample of clusters only near its completion. In these initial stages of the survey, however, studies the abundance pattern of individual clusters are very valuable, not only to test methods and tools, but also because, as mentioned, few young clusters so far have solid abundance determination. Adding information and statistics it is hence very important.

In particular, in this paper we present the products released internally to the Gaia-ESO Survey consortium on the first observed PMS cluster: gamma Velorum. The cluster properties, the target selection and spectral analysis are detailed in Sect. 2. The comparison between the main stellar parameters derived with the two different spectrographs, UVES and Giraffe, is given in Sect. 3. The identification of the cluster members is presented in Sect. 4, while the results of the elemental abundance determination are discussed in Sect. 5. Finally, the conclusions are outlined in Sect. 6.

\section{Observations and data processing}

The work presented in this paper is based on the results of the analysis of the spectra obtained during the first six months of observations (January-June 2012) and released internally in the GESviDR1Final catalog (August 2013). In the following, we describe the properties of gamma Velorum, the target selection, the observations, and the spectroscopic analysis. 


\subsection{The gamma Velorum open cluster}

Gamma Velorum is a nearby $(\sim 350 \mathrm{pc})$ open cluster for which Jeffries et al. (2009) originally claimed an age of 5-10 Myr, but that could instead be older than $10 \mathrm{Myr}$ (but younger than $20 \mathrm{Myr}$; see discussion in Jeffries et al. 2014). Its low-mass members are distributed around a double-lined spectroscopic binary system (hereafter $\gamma^{2}$ Vel, as in Jeffries et al. 2009), composed of a Wolf-Rayet (hereafter WR) WC8 star (the closest Wolf-Rayet star to the Sun; Smith 1968) and an O8 massive star (Schaerer et al. 1997). Pozzo et al. (2000) first recognized the presence of low-mass stars around the more massive objects. Because of the low extinction and reddening $\left(A_{V}=0.131\right.$ and $E_{B-V}=0.038$; Jeffries et al. 2009), the sparse disk population and youth of the gamma Velorum association, the sequence of the cluster is clearly visible in the optical color-magnitude diagrams (CMDs) presented by Jeffries et al. (2009).

On a larger scale gamma Velorum lies in the so-called Vela complex (see Pettersson 2008; Sushch et al. 2011), a very composite region characterized, inter alia, by the presence of a number of PMS clusters (e.g., gamma Velorum, $\operatorname{Tr} 10$, and NGC 2547), three OB associations (Humphreys 1978; Brandt \& Maran 1971; Slawson \& Reed 1988) and two supernova remnants (the Gum nebula and the Vela SNR). The latter have been created by two or more supernovae explosions that occurred 1-6 Myr and 11400 yr ago (Pettersson 2008). The shocks from the latter SN have not yet reached the gamma Velorum cluster (Sushch et al. 2011), but it is clear that the environment has been subject to a fast dynamical evolution. In this context, the analysis of the Gaia-ESO Survey data has led Jeffries et al. (2014) to conclude that 208 members of the gamma Velorum cluster, targeted by Giraffe and identified through their lithium content, are grouped in two distinct kinematic populations. More specifically, through a maximum-likelihood fit of the RV distribution they have found that the first kinematic component (population A), centered at $\mathrm{RV}_{1}=16.70 \mathrm{~km} \mathrm{~s}^{-1}$, is narrower and consistent with virial equilibrium $\left(\sigma_{1}=0.28 \mathrm{~km} \mathrm{~s}^{-1}\right)$, while the second component (population B) is much broader $\left(\sigma_{2}=1.85 \mathrm{~km} \mathrm{~s}^{-1}\right)$ and centered at higher velocities, i.e., $\mathrm{RV}_{2}=18.58 \mathrm{~km} \mathrm{~s}^{-1}$.

Interestingly, $\gamma^{2}$ Vel appears to be younger than the low-mass stars. Indeed, the relation and interactions of $\gamma^{2}$ Vel with the lowmass cluster members is still debated, mainly because of the age of the central WR star. In fact, even if the most recent $\gamma^{2}$ Vel distance determinations $368_{-13}^{+38} \mathrm{pc}$ (Millour et al. 2007), 336 $6_{-7}^{+8} \mathrm{pc}$ (North et al. 2007) and $334_{-40}^{+32}$ pc (van Leeuwen 2007) support its association with the cluster, the age estimates of $3.5 \pm 0.4 \mathrm{Myr}$ (North et al. 2007), and $5.5 \pm 1$ Myr (Eldridge 2009) indicate that $\gamma^{2}$ Vel is younger than the majority of the low-mass members of the cluster.

In spite of the remarkable properties of the cluster and of the Vela complex which makes gamma Velorum a suitable target for a spectroscopic survey, its iron abundance is still unknown. Gaia-ESO Survey observations hence allow us to perform the first abundance study of this cluster.

\subsection{Target selection and observations}

The Gaia-ESO Survey observations are performed with the multi object optical spectrograph FLAMES on the VLT (Pasquini et al. 2002). This instrument makes use of two spectrographs, Giraffe (132 fibers) and UVES (eight fibers).

We based the target selection criteria on homogeneous photometric data, covering a large area of the cluster field. In

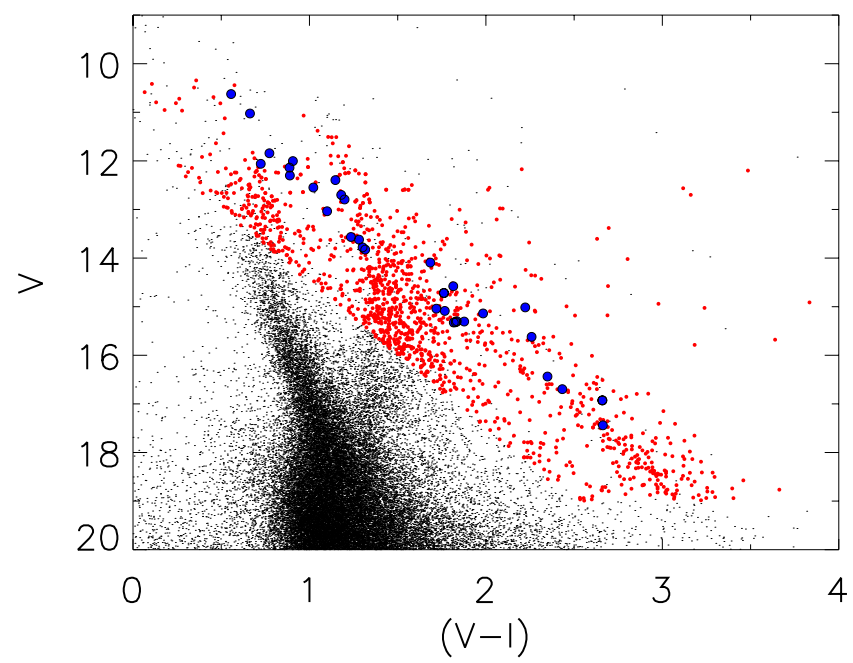

Fig. 1. Color-magnitude diagram of the 1283 stars observed in gamma Velorum (in red), along with all the stars (in black) located in the field of view centered on $\gamma^{2}$ Vel with photometry reported in Jeffries et al. (2009). The known members from this paper are shown in blue.

particular, we chose the list of targets considering only the sources within a region of 0.9 square degrees centered on $\gamma^{2} \mathrm{Vel}$ and studied by Jeffries et al. (2009). We selected these targets mostly following the guidelines for cluster observations (see Bragaglia et al., in prep.).

The final sample was chosen so as to include all photometric candidate members in a region of the CMD around the cluster sequence, defined by previously known members. We considered all stars falling within \pm 1.5 mag of the cluster sequence as high priority targets. A few lower priority stars have also been targeted to use spare fibers. The CMD of the selected sources is shown in Fig. 1. The cluster sequence identified by Jeffries et al. (2009) is clearly visible as the upper concentration of red dots with $(V-I) \sim 1.8-3.2$. The spectroscopic survey is limited to $V \lesssim 13.5$ mag and $V \lesssim 19$ mag for UVES and Giraffe. Further discussion of target selection can also be found in Jeffries et al. (2014).

A total of 18 fields, forming a mosaic around $\gamma^{2}$ Vel, were completed during runs A (nights from 2011-12-31 to 2012-01-02) and B (night 2012-02-12) of P88, using the CD\#3 cross-disperser $(\lambda=4770-6820 \AA ; R=47000)$ for UVES and the HR15N grating $(\lambda=6440-6820 \AA ; R \sim 17000)$ for Giraffe. Each field was observed for either $20 \mathrm{~min}$ (nine fields) or $50 \mathrm{~min}$ (nine fields). The exposure times hence vary from $20 \mathrm{~min}$ for brighter stars $(V \leq 12$ and $V \leq 16$ for UVES and Giraffe, respectively) to $50 \mathrm{~min}$ for fainter stars $(12<V<13.5$ and $16<V<19)$. Stars lying in overlapping fields have longer exposure times. We acquired spectra for a total of 1242 and 80 individual stars with Giraffe and UVES, respectively. We observed 39 stars with both spectrographs. Signal-to-noise ratios (S/Ns) for the UVES spectra are in the range 20-300, with a median value of 116 . The Giraffe spectra have S/Ns ranging between 3 and 300 with a median of 84 .

\subsection{Available data}

The Gaia-ESO Survey is structured in 20 working groups (WGs) dedicated to different tasks. Data reduction and determination of radial velocities (RVs) and projected rotational velocities are carried out, independently for Giraffe and UVES, by two different 
Table 1. Stellar parameters of the 80 UVES targets.

\begin{tabular}{ccccccc}
\hline \hline ID & Cname & $\begin{array}{c}\text { RA } \\
(\mathrm{J} 2000)\end{array}$ & $\begin{array}{c}\text { Dec } \\
(\mathrm{J} 2000)\end{array}$ & $\begin{array}{c}T_{\text {eff }} \\
(\mathrm{K})\end{array}$ & $\begin{array}{c}\log g \\
(\mathrm{dex})\end{array}$ & $\begin{array}{c}{[\mathrm{Fe} / \mathrm{H}]} \\
(\mathrm{dex})\end{array}$ \\
\hline 1 & $08063616-4748206$ & 080636.16 & -474820.6 & $6726 \pm 347$ & $4.16 \pm 0.21$ & $-1.51 \pm 0.20$ \\
2 & $08064772-4659492$ & 080647.72 & -465949.2 & $5776 \pm 49$ & $4.20 \pm 0.08$ & $-0.02 \pm 0.03$ \\
$\ldots$ & $\ldots$ & $\ldots$ & $\ldots$ & $\ldots$ & $\ldots$ & $\ldots$ \\
\hline
\end{tabular}

Notes. The full version is available at the CDS.

Table 2. Quantities used for the membership analysis of the UVES sample.

\begin{tabular}{cccccccccc}
\hline \hline ID & $\begin{array}{c}\mathrm{RV} \\
\left(\mathrm{km} \mathrm{s}^{-1}\right)\end{array}$ & $\begin{array}{c}E W(\mathrm{Li}) \\
(\mathrm{m} \AA)\end{array}$ & $\begin{array}{c}(B-V)_{0} \\
(\mathrm{mag})\end{array}$ & $\begin{array}{c}V_{0} \\
(\mathrm{mag})\end{array}$ & $\begin{array}{c}\mathrm{RV} \\
\mathrm{mem} .\end{array}$ & $\begin{array}{c}\log g \\
\text { mem. }\end{array}$ & $\begin{array}{c}\mathrm{Li} \\
\text { mem. }\end{array}$ & $\begin{array}{c}\text { CMD } \\
\text { mem. }\end{array}$ & $\begin{array}{c}\text { Final } \\
\text { mem. }\end{array}$ \\
\hline 1 & 13.5 & $<20$ & 0.13 & 1.97 & $\mathrm{~N}$ & $\mathrm{Y}$ & $\mathrm{HCM}$ & $\mathrm{N}$ & $\mathrm{N}$ \\
2 & 10.4 & $<10$ & 0.64 & 4.99 & $\mathrm{~N}$ & $\mathrm{Y}$ & $\mathrm{N}$ & $\ldots$ & $\mathrm{N}$ \\
$\ldots$ & $\ldots$ & $\ldots$ & $\ldots$ & $\ldots$ & $\ldots$ & $\ldots$ & $\ldots$ & $\ldots$ & $\ldots$ \\
\hline
\end{tabular}

Notes. The table shows that we have identified eight high-probability members and eight HCMs (see text). RV values are not corrected for the $1.1 \mathrm{~km} \mathrm{~s}^{-1}$ systematic shift. The full version is available at the CDS.

teams of WG7. The Giraffe data are reduced using a pipeline, specifically developed for the Gaia-ESO survey, which performs the basic steps of the data reduction process (i.e., bias subtraction, flat-fielding, spectra extraction, and wavelength calibration), sky subtraction, and calculation of preliminary RVs and projected rotational velocities, by cross-correlating the spectra with a grid of templates. To improve the precision of the RVs and projected rotational velocities, we fitted the reduced spectra with a low-order polynomial multiplied by a template spectrum. The RV, the projected rotational velocities, the polynomial coefficients and the template parameters (temperature, gravity and metallicity) are free parameters of the fit, with initial guesses derived by the first pipeline. We reduced the UVES data using the FLAMES-UVES ESO public pipeline. A specific pipeline developed for the Gaia-ESO Survey is used for the sky subtraction and the calculation of RVs and projected rotational velocities, by cross-correlating each spectra for a grid of templates. A more detailed discussion of the procedures used for the data reduction, and the calculation of RV, and projected rotational velocities is reported in Lewis et al. (in prep.) and Jeffries et al. (2014) for Giraffe, and in Sacco et al. (2014) for UVES.

As for spectrum analysis, WG11 (including the contribution of up to 13 nodes) is dedicated to the analysis of the UVES spectra of F-G-K stars, while WG12 (composed by four nodes) focuses on young stars, analyzing both UVES and Giraffe spectra. The analysis performed by WG11 and WG12 is described in detail in Smiljanic et al. (2014) and Lanzafame et al. (in prep.), respectively. Whereas we will briefly describe here how the recommended parameters released to the consortium are derived, we refer to the above two papers for a full description of the approach and methodologies.

Both WG11 and WG12 benefit from the contribution of nodes that use different methods of analysis. These different approaches can be summarized as follows: i) nodes that employ the equivalent width (EW) analysis; the atmospheric parameter determination is based on the excitation and ionization balance of the iron lines; ii) nodes that use spectrum synthesis and estimated atmospheric parameters from a $\chi^{2}$ fit to observed spectra; in some cases the grid of templates is composed by observed spectra of slow-rotating, low-activity stars; iii) multi linear regression methods that simultaneously determine the stellar parameters of an observed spectrum by the projection of the spectrum onto vector functions, constructed as an optimal linear combination of the local synthetic spectra. The parameters released in GESviDR1 are obtained by each of the two working groups by computing the median value of the results provided by the nodes, after the outliers have been discarded. Uncertainties are the node-to-node dispersions. We mention that the consortium uniformly makes use of MARCS models of stellar atmospheres (Gustafsson et al. 2008) that assume the solar abundances from Grevesse et al. (2007). Also, common atomic data have been used for the analysis of all the spectra of the Gaia-ESO Survey. Similarly, more than one node measure the strength of the Li I line at $6707.8 \AA$ in both Giraffe and UVES spectra. The nodes use independent methods to derive the EW of this features: specifically, some of them apply a Gaussian fitting to the line, while others are based on the direct profile integration of the line. The median value of the EW (or the average, when only two nodes provided the measurement) are then adoped. All these procedures are detailed in Lanzafame et al. (in prep.).

Released parameters for gamma Velorum include radial and rotational velocities, CCFs and the products of the spectrum analysis. The latter include the main atmospheric parameters ( $T_{\mathrm{eff}}, \log g$, and $\left.[\mathrm{Fe} / \mathrm{H}]\right)$ and other parameters (e.g., veiling, strength of the Li I line at $6707.8 \AA$, H $\alpha$, etc.), along with their uncertainties. All our UVES targets, along with their with RVs and parameters, when available, are listed in Tables 1 and 2. Individual elemental abundances are also provided for UVES spectra, whenever they can be measured. The first four rows of Table 5 represent a brief outline of the data obtained from the UVES spectra.

To summarize:

- 80 UVES and 1242 Giraffe targets observed in the gamma Velorum fields;

- we found six UVES targets to be double-lined binaries (SB2; see Sect. 4). In the Gaia-ESO catalog, RV values are available for all of these systems. Also, the main parameters of one SB2 have been delivered by the consortium.

- RV estimates are available for 73 of the UVES targets; hereafter we disregard the RV values of five UVES targets either with poor quality spectra or that are early-type stars or fast rotating sources $\left(v \sin i>100 \mathrm{~km} \mathrm{~s}^{-1}\right)$, and hence the inferred radial velocities are highly uncertain. Moreover the RV values of the six SB2 observed with UVES are not considered. Thus, the final sample of stars with available and reliable RV 
Table 3. Stellar parameters of the 39 stars targeted by both UVES and Giraffe.

\begin{tabular}{lccc}
\hline \hline Star & $\begin{array}{c}T_{\text {eff }} \\
(\mathrm{K})\end{array}$ & $\begin{array}{c}\log g \\
(\mathrm{dex})\end{array}$ & $\begin{array}{c}{[\mathrm{Fe} / \mathrm{H}]} \\
(\mathrm{dex})\end{array}$ \\
\hline $08064772-4659492$ & $5765 \pm 70$ & $4.09 \pm 0.27$ & $-0.19 \pm 0.11$ \\
$08065592-4704528$ & $4436 \pm 57$ & $2.48 \pm 0.28$ & $-0.11 \pm 0.02$ \\
$\ldots$ & $\ldots$ & $\ldots$ & $\ldots$ \\
\hline
\end{tabular}

Notes. The full version is available at the CDS.

estimates consists of 62 UVES stars. The RV values for the UVES sample are listed in Table 2. We refer to Jeffries et al. (2014) for the RV estimates of the Giraffe targets;

- A measurement or an upper-limit of the EW of the Li I line is available for all stars with the exception of four warm stars in the UVES sample that do not show any Li feature in their spectra. For these four stars, we assume a $3 \sigma$ detection upper-limit using the Cayrel formula (Cayrel 1988). These values are listed in Table 2. As for the RV values, we also refer to Jeffries et al. (2014) for the Li equivalent width measurements in the Giraffe spectra;

- After the rejection of the main parameters derived for the SB2 target, 67 stars observed with UVES have an estimate of the fundamental parameters. Note that these are available for 36 of 39 stars observed with both spectrographs. The main parameters for all the UVES targets are listed in Table 1, those obtained from Giraffe spectra for stars observed with both spectrographs are listed in Table 3, while the main parameters of the Giraffe members identified by Jeffries et al. (2014) are listed in Table 4. The mean uncertainties of the parameters derived from UVES spectra are: $\left\langle\sigma_{T_{\text {eff }}}\right\rangle=120 \mathrm{~K},\left\langle\sigma_{\log g}\right\rangle=0.17 \mathrm{dex},\left\langle\sigma_{[\mathrm{Fe} / \mathrm{H}]}\right\rangle=0.10 \mathrm{dex}$;

- Finally, individual elemental abundances have been derived for 47 stars observed with UVES.

Note that a few stars with atmospheric parameters do not have an RV estimate and vice versa.

\section{UVES vs. Giraffe}

As mentioned, atmospheric parameters and $[\mathrm{Fe} / \mathrm{H}]$ values have been also released for the Giraffe targets, however, since the analysis of high-resolution spectra should yield more reliable iron abundance values (see Sect. 5.1), most of our scientific analysis will focus on the results of the UVES observations. On the other hand, we will mostly use the Giraffe sample as a control sample to infer the membership of the UVES targets; therefore, in this section we take advantage of the stars observed with both spectrographs to check for the consistency of the inferred parameters. In particular, we will make a detailed comparison of the RVs, lithium EWs, and atmospheric stellar parameters $\left(T_{\mathrm{eff}}\right.$, $\log g$, and $[\mathrm{Fe} / \mathrm{H}])$.

\subsection{Radial velocities}

In Fig. 2, we show the difference between the values of the RV as a function of the projected rotational velocity derived from the UVES spectra. In the case of UVES, we adopt as final RV the mean of the two values obtained using the upper and lower spectral regions. As for the error bars, we assume the largest value between the error quoted in the survey catalog $\left( \pm 0.6 \mathrm{~km} \mathrm{~s}^{-1}\right)$ and the difference between the RVs measured independently in

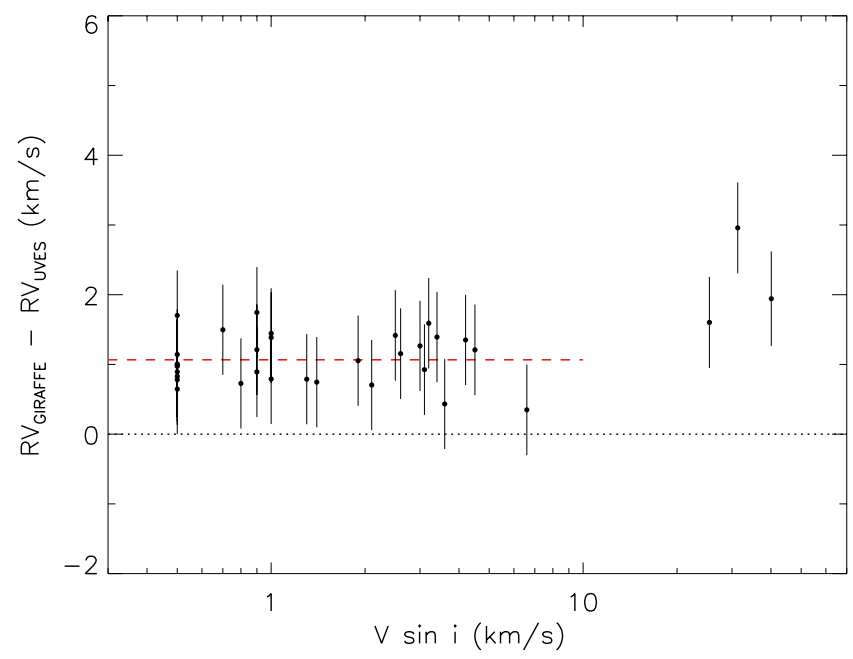

Fig. 2. Comparison of the RV of the 34 stars with both Giraffe and UVES spectra and available RV values. The difference of the RVs is plotted as a function of the stellar rotational velocity $(v \sin i)$. The red dashed line represents the offset between $\mathrm{RV}_{\text {Giraffe }}$ and $\mathrm{RV}_{\mathrm{UVES}}$ for the 31 stars with $v \sin i<10 \mathrm{~km} \mathrm{~s}^{-1}$.

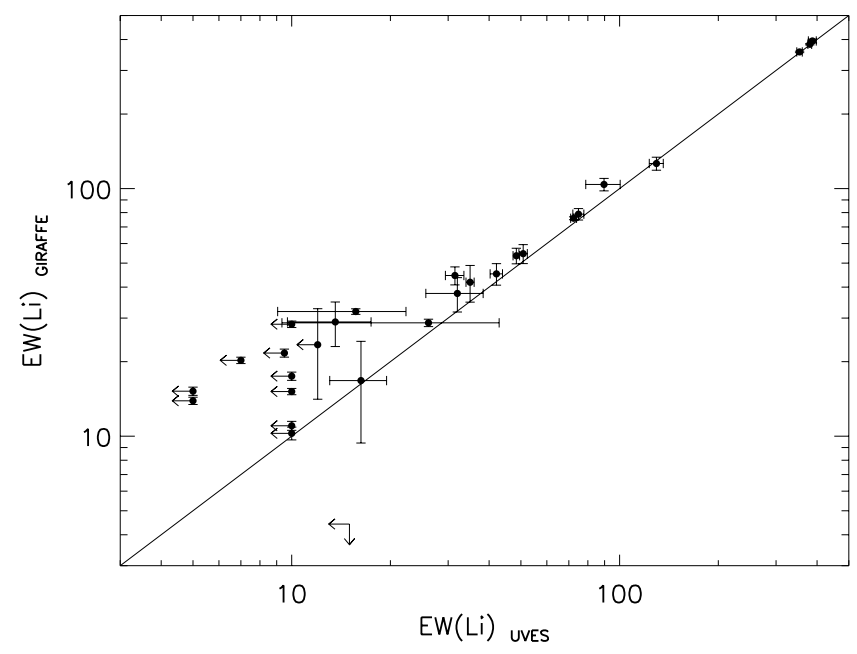

Fig. 3. Comparison of the $17 \mathrm{EW}(\mathrm{Li})$ measurements obtained from both the Giraffe and UVES spectra.

the two CCDs (Sacco et al. 2014). We note that $\mathrm{RV}_{\text {Giraffe }}$ is systematically higher than $\mathrm{RV}_{\mathrm{UVES}}$ by $1.1 \pm 0.4 \mathrm{~km} \mathrm{~s}^{-1}$ (red dashed line in Fig. 2) up to about $v \sin i=10 \mathrm{~km} \mathrm{~s}^{-1}$ and the difference increases for larger rotational velocities. While the origin of this offset needs further investigation (see Sacco et al. 2014), for the time being we applied an offset of $+1.1 \mathrm{~km} \mathrm{~s}^{-1}$ to UVES RVs.

\subsection{Li equivalent widths}

In Fig. 3 we show a comparison of the EW of the Li I $6707.8 \AA$ line measured in Giraffe and UVES spectra, respectively. The figure indicates a very good agreement for most of the stars down to about $30 \mathrm{m \AA}$; a discrepancy between the values is instead present below that value, where the Giraffe measurements are systematically higher than the UVES measurements. This difference needs to be further investigated and may be related to the different resolving powers and the blending with the nearby Fe I 6707.4 A line; however, we stress that it will not 
Table 4. Iron abundances of the 208 members targeted by Giraffe.

\begin{tabular}{lccccc}
\hline \hline Star & $\begin{array}{c}\text { RA } \\
(\mathrm{J} 2000)\end{array}$ & $\begin{array}{c}\text { Dec } \\
(\mathrm{J} 2000)\end{array}$ & $\begin{array}{c}T_{\text {eff }} \\
(\mathrm{K})\end{array}$ & $\begin{array}{c}\log g \\
(\mathrm{dex})\end{array}$ & $\begin{array}{c}{[\mathrm{Fe} / \mathrm{H}]} \\
(\mathrm{dex})\end{array}$ \\
\hline $08064077-4736441$ & 080640.77 & -473644.1 & $4798 \pm 98$ & $2.77 \pm 0.25$ & $-0.18 \pm 0.02$ \\
$08064390-4731532$ & 080643.90 & -473153.2 & $3259 \pm 60$ & $4.76 \pm 0.15$ & $-0.32 \pm 0.17$ \\
$\ldots$ & $\ldots$ & $\ldots$ & $\ldots$ & $\ldots$ & $\ldots$ \\
\hline
\end{tabular}

Notes. The full version is available at the CDS.
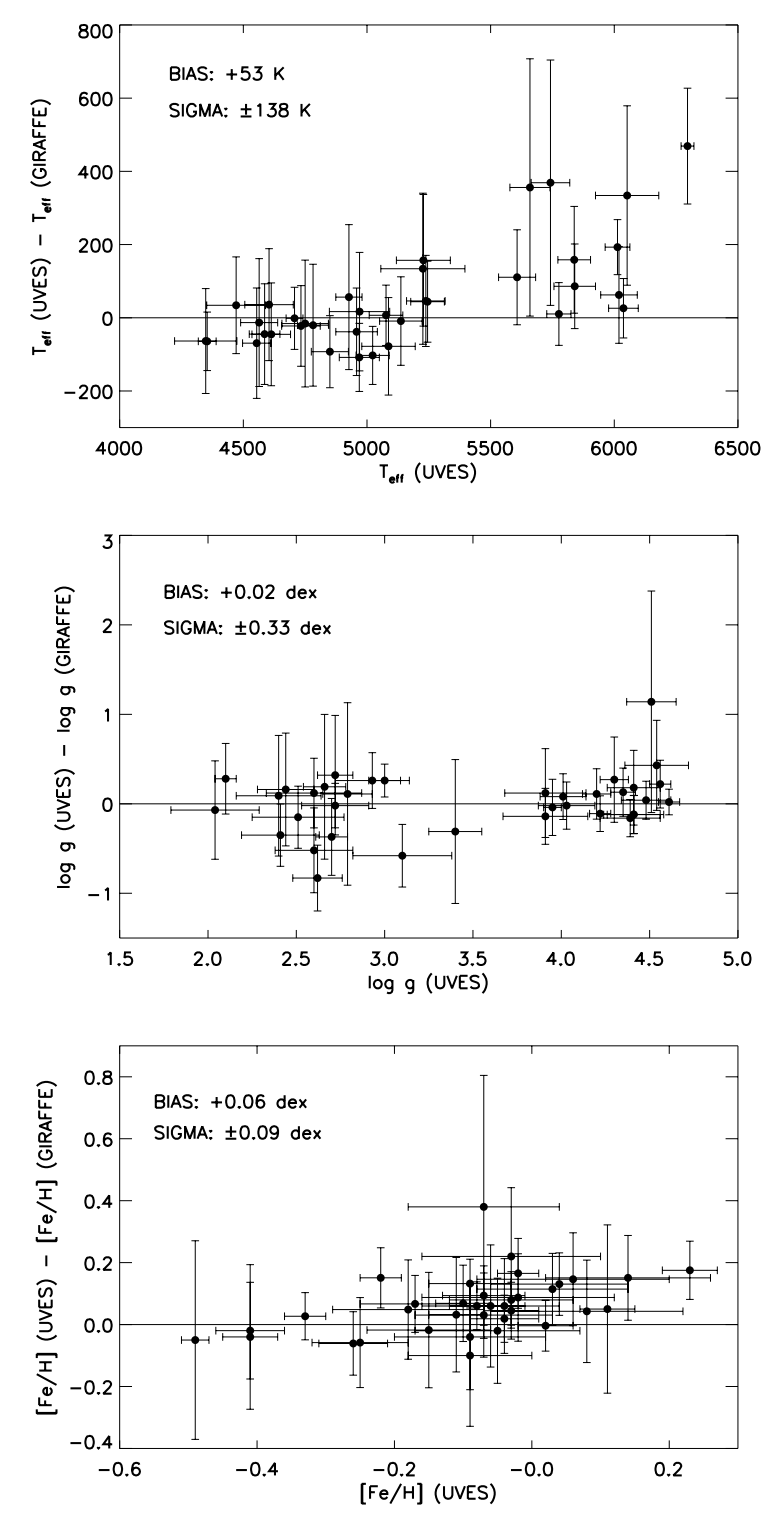

Fig. 4. Comparison of the stellar parameters of the stars observed with both Giraffe and UVES. From top to bottom, we show the effective temperature, the surface gravity, and the iron abundance. Systematic biases and standard deviations are reported in each panel.

affect our discussion and conclusions on lithium membership, since the threshold between Li members and nonmembers is set at higher values of the EWs (see Sect. 4.3).

\subsection{Atmospheric parameters}

In Fig. 4, we compare the fundamental parameters derived from Giraffe and UVES analyses for 36 of the 39 stars observed with both instruments. As in the case of $\mathrm{EW}(\mathrm{Li})$, we conclude that the two spectrographs yield compatible values within the errors for the majority of the stars. The only discrepancy is seen for the effective temperature of warm stars $\left(T_{\text {eff }}>5500 \mathrm{~K}\right)$ for which the Giraffe analysis gives somewhat lower values than UVES, but is still marginally consistent with them. Again, the origin of these differences is under investigation, but it does not affect our conclusions on UVES membership. Also note that, because of the lower resolution and the shorter spectral range, the uncertainties on the data derived from Giraffe are larger. This widens the scatter of the data without a significant implication for our analysis.

To summarize, whereas we will account for the offset between Giraffe and UVES in the following RV membership analysis, no systematic biases are present for lithium and $\log g$ values, the additional two criteria that we will use for confirming the membership of UVES candidates.

\section{Membership analysis}

In this section, we will use the spectroscopic information, specifically, RVs, the strength of the Li line, and the stellar surface gravity, along with the position of the targets in the CMD, to perform the membership analysis of the UVES targets. In Table 5, we summarize each step of the selection procedure that, starting from the 80 stars observed with UVES, leads to a restricted sample of high-probability members that will be used for the metallicity analysis, which is the main goal of the present paper.

As a first step, we searched for the presence of spectroscopic binaries in the sample of UVES stars. We identify six double-lined binaries (SB2) through their released CCFs: namely, J08072516-4712522, J08073722-4705053, J080935894718525, J08103996-4714428, J08105382-4719579, and J08115305-4654115. Those systems were hence discarded from the sample analyzed for membership. Note that for one of the SB2 systems fundamental parameters are available.

\subsection{Radial velocity distribution}

We have considered all UVES candidates with available RV and that have not been identified as SB2 systems for the radial velocity analysis. This adds up to 62 stars. Also, for the estimate of the RV membership, we have added $1.1 \mathrm{~km} \mathrm{~s}^{-1}$ to the RVs from UVES spectra to account for the systematic offset with respect to Giraffe described in Sect. 3.1.

Assuming that the UVES targets would be characterized by the same RV distribution as the Giraffe targets, the analysis was performed adopting the results of Jeffries et al. (2014); specifically, considering the two kinematic components identified in that study, along with their peak velocity and dispersion, we defined as RV members all the stars with RVs in the interval between 14.9 and $22.3 \mathrm{~km} \mathrm{~s}^{-1}$, corresponding to $\mathrm{RV}_{2} \pm 2 \sigma_{2}$ of the broader distribution. Among the 62 UVES candidates, we have 
Table 5. Summary of the selection procedure of UVES candidate members.

\begin{tabular}{|c|c|c|}
\hline Observed & 80 candidates & 39 in common with Giraffe \\
\hline Binaries & $6 \mathrm{SB} 2$ & discarded from the sample \\
\hline RV estimates & 62 candidates & $\begin{array}{l}7 \mathrm{RV} \text { members } \\
55 \mathrm{RV} \text { nonmembers (or SB1) }\end{array}$ \\
\hline Fundamental parameters & 67 candidates & $\begin{array}{l}37 \mathrm{MS} / \mathrm{PMS} \text { stars } \\
30 \text { giants }\end{array}$ \\
\hline $\begin{array}{l}\mathrm{EW}(\mathrm{Li}) \\
\text { excluding giants }\end{array}$ & 74 candidates -30 (giants) $=44$ & $\begin{array}{l}8 \mathrm{Li} \text { members } \\
19 \mathrm{HCM}^{*} \text { (8 rejected as RV nonmembers) } \\
17 \mathrm{Li} \mathrm{nonmembers}^{\text {Linem }}\end{array}$ \\
\hline CMD & $8($ Li members $)+11(\mathrm{HCM})=19$ & $\begin{array}{l}7 \text { Li mem. consistent with ZAMS } \\
1 \text { Li mem. below ZAMS } \\
5 \text { HCM consistent with ZAMS } \\
6 \text { HCM below ZAMS (rejected as nonmembers) }\end{array}$ \\
\hline $\begin{array}{l}\text { Abundance } \\
\text { analysis }\end{array}$ & $\begin{array}{l}8 \mathrm{Li} \text { mem with }[\mathrm{Fe} / \mathrm{H}] \text { values } \\
2 \mathrm{Li} \mathrm{mem} \text { with other elements estimates }\end{array}$ & \\
\hline
\end{tabular}

Notes. ${ }^{(*)}$ In the text we use "Hot Candidate Members" $(\mathrm{HCMs})$ for the stars with $(B-V)_{0}<0.35$ for which we cannot use lithium as a membership criterion.

identified seven RV members and 55 stars whose RV values lie out from the $\mathrm{RV}_{2} \pm 2 \sigma_{2}$ boundaries. Indeed, some of these stars can be binary systems that are members of gamma Velorum, however, hereafter, we will refer to this stars as RV nonmembers.

\subsection{Identification of the giant contaminants}

The sequence of gamma Velorum candidate members is easily identified in optical CMDs at magnitudes $V>15 \mathrm{mag}$ (see Jeffries et al. 2009), however, the UVES targets are restricted to the brighter part of the CMD where the sequence is heavily contaminated by field stars. In order to identify the population of evolved star contaminants, we plot in Fig. 5 the spectroscopic surface gravity as a function of $T_{\text {eff }}$ for the UVES targets along with the Giraffe targets identified as cluster members by Jeffries et al. (2014). Stars are clearly divided in two groups: mainsequence and PMS stars with $\log g$ between 4 and 5 dex, and giant stars with lower gravity values. In the figure, we also show the $5 \mathrm{Myr}$ (solid line), 1 and $10 \mathrm{Myr}$ (dashed lines) isochrones from Siess et al. (2000) models for a metallicity of $Z_{\odot}=0.01$, close to the value of the solar metallicity, $Z=0.012$, adopted in the MARCS models. Based on this figure, we conservatively consider all the UVES stars that lie above the $5 \mathrm{Myr}$ isochrone as giant contaminants. Using this criterion we find that out of the 67 non-SB2 systems with log $g$ determination, 37 lie below the 5 Myr isochrone: the seven RV members, 22 candidates with RV not consistent with that of the cluster, and eight stars without an RV estimate. The remaining 30 UVES stars, with log $g$ typical of a giant star will be discarded from further analysis. Not surprisingly, all these stars are RV nonmembers.

\subsection{Lithium members}

As is well known, lithium is amongst the most useful membership indicator for young stars. In Fig. 6, we show the EW(Li) as a function of the intrinsic color $(B-V)_{0}$ for the 44 UVES candidates that have not been rejected as SB2 systems or giant contaminants or do not have any $\log g$ measurement. We derived the intrinsic $B-V$ colors from the photometry reported in Jeffries et al. (2009) dereddened adopting the $E_{B-V}$ estimated for gamma Velorum by the same authors. Along with the UVES stars, we also plot the 208 Giraffe targets classified as

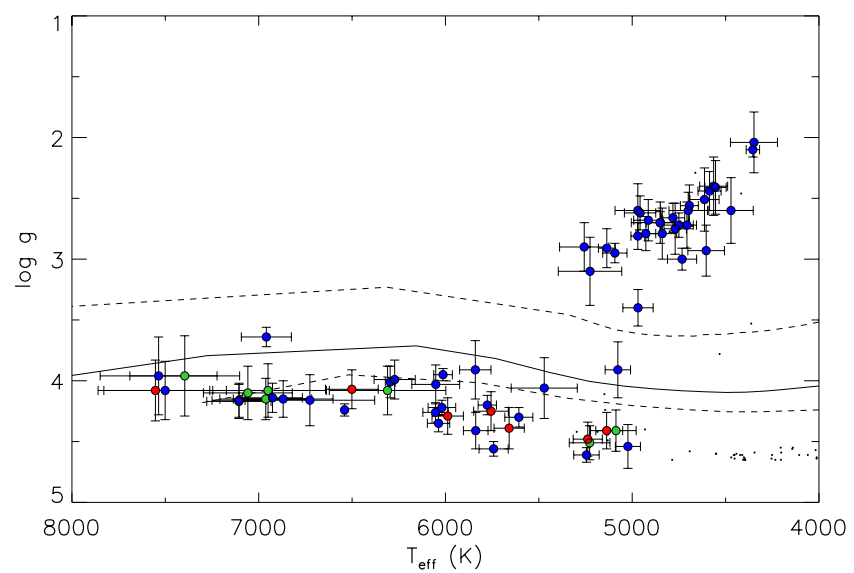

Fig. 5. Surface gravity versus effective temperature. The diagram allows us to identify the giant field stars in the UVES sample as the objects that lie above the $5 \mathrm{Myr}$ isochrone (solid line) together with the 1 and $10 \mathrm{Myr}$ (dashed lines) isochrones using Siess et al. (2000) models. The different colors indicate RV members (red), RV nonmembers (blue), and stars excluded from the RV analysis (green). The black dots show the Giraffe members identified by Jeffries et al. (2014). Note that a few Giraffe Li members have gravity values below the $10 \mathrm{Myr}$ isochrone. The diagram is limited to the temperature range relevant for the UVES targets.

cluster members by Jeffries et al. (2014). Most of the Giraffe targets are in the color range $0.7<(B-V)_{0}<1.3$ and have $E W(\mathrm{Li})>200 \mathrm{~m} \AA$. Their distribution clearly defines the sequence of $\mathrm{Li}$ undepleted members. At $(B-V)_{0}>1.3$, however, we observe a large dispersion in equivalent widths, indicating that a fraction of low-mass stars in the cluster have started depleting lithium and hence suggesting a possible age dispersion (see Jeffries et al. 2014; Franciosini et al., in prep.).

In order to assess the membership of the UVES sources on the basis of the lithium content, we also use the available information for the members of the Pleiades cluster ( 125-130 Myr; Stauffer et al. 1998), similar to the approach of James et al. (2006). The comparison of the EWs(Li) of our sources with those of Pleiades members with similar $(B-V)_{0}$ will allow us to identify the youngest targets, which are therefore the likely members of gamma Velorum. Among the UVES targets in the 


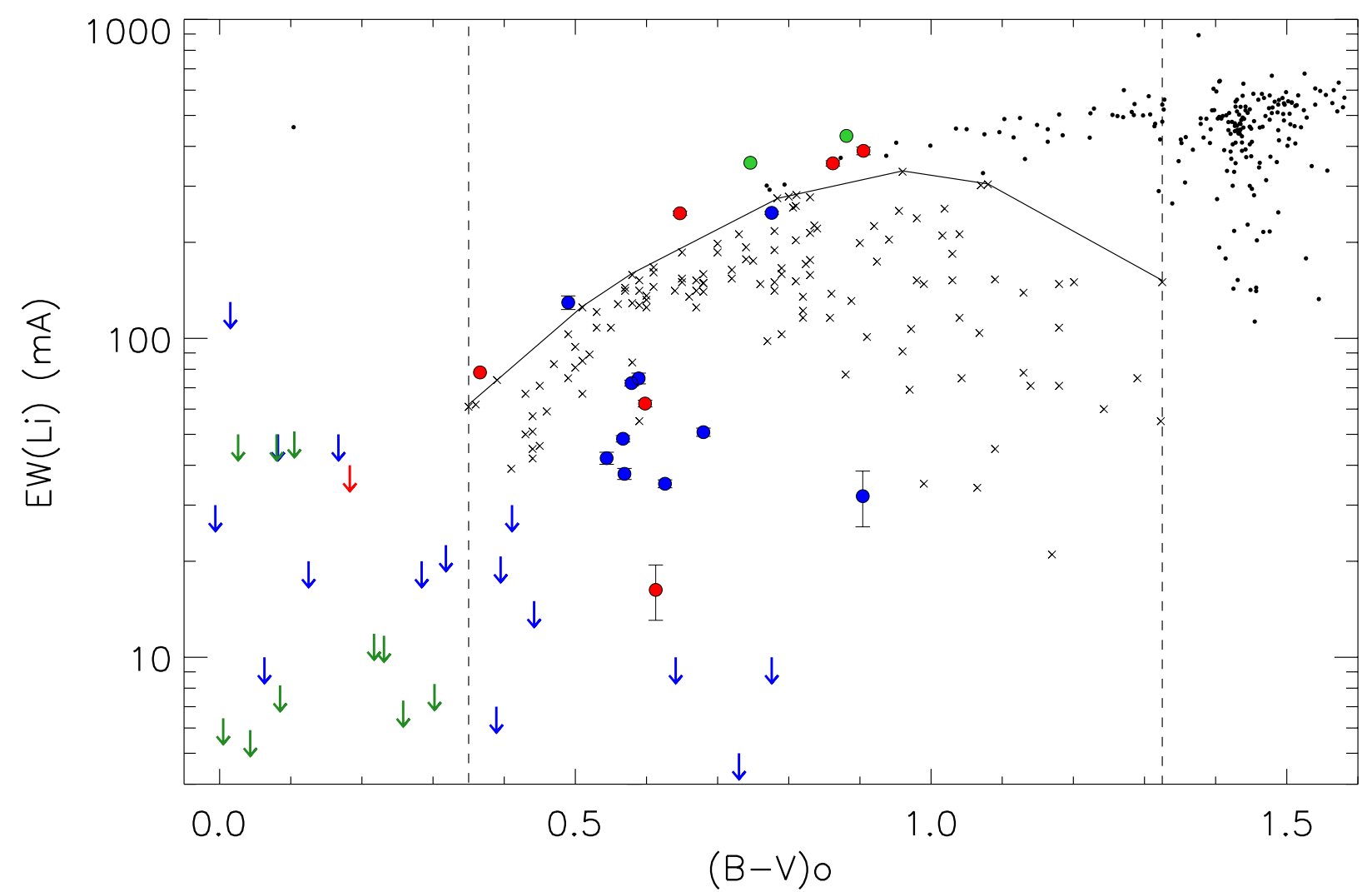

Fig. 6. Lithium EW as a function of the intrinsic color $(B-V)_{0}$. The red, blue, and green symbols (circles and arrows) represent the RV members, RV nonmembers, and stars with no RV estimate from the UVES sample, respectively. Most of the Li detections in UVES spectra have uncertainties associated with their EWs that are smaller than the data points. The black dots show the Giraffe members identified by Jeffries et al. (2014). Their uncertainties, which have typical values of $\pm 10-20 \mathrm{~m} \AA$, are not represented in the plot. Also, the uncertainties associated with the colors are not plotted, since their typical values of \pm 0.02 are negligible. The solid line denotes the upper envelope of the Pleiades distribution (crosses; Soderblom et al. 1993; Jones et al. 1996). The dashed lines identify the color range $0.35<(B-V)_{0}<1.33$ spanned by the Pleiades members.

range of colors spanned by the Pleiades members seven stars have $E W(\mathrm{Li})$ higher than their Pleiades counterparts, since they lie above the upper envelope of the Pleiades Li-color distribution. One additional UVES target, with $E W(\mathrm{Li})>200 \mathrm{~m} \AA$, lies slightly below the upper envelope of the Pleiades. All of the other UVES stars have $E W(\mathrm{Li})<100 \mathrm{~m} \AA$ and are located significantly below the Pleiades distribution. Most of these latter stars are RV nonmembers and their low lithium suggests that they are not associated with the gamma Velorum cluster and are likely field contaminants. On the other hand, the seven stars with EW(Li) greater than the upper envelope of the Pleiades distribution are substantially younger than the Pleiades, thus are likely members of gamma Velorum. The case of the star lying slightly below the upper envelope is less obvious and its membership needs to be further assessed on the basis of a CM diagram in Sect. 4.4. We note that this star is an RV nonmember, hence a possible binary member. To conclude, we consider all of the stars with $0.35<(B-V)_{0}<1.33$ lying above the upper envelope of the Pleiades distribution, plus the UVES target lying slightly below that limit as Li members/candidates. For stars with $(B-V)_{0}<0.35$, we cannot use lithium as a membership criterion, but we flagged them as "hot candidate members" (hereafter, HCM). The membership of all these stars will be further checked in Sect. 4.4.

To summarize, the analysis of $\mathrm{Li}$ allows us to conclude that there are eight high-probability UVES Li members (four RV members, two RV nonmembers, and two stars without an $\mathrm{RV}$ estimate). Fifteen RV nonmembers appear to be older than the Pleiades counterparts, thus likely contaminants. Also two RV members have small EW(Li) and hence appear to be nonmembers based on their lithium content. As for the hotter stars, there are $11 \mathrm{HCMs}$ on the left side of the dashed line (one RV member, ten without RV estimate) that we will consider for further analysis and eight HCMs that are RV nonmembers, which will be rejected. Interestingly, we also note that one of the six SB2 systems (J08093589-4718525, \#46) has both components with $\mathrm{EW}(\mathrm{Li})$ larger than $100 \mathrm{~m} \AA$, making it a possible member of the cluster.

\subsection{Color-magnitude diagram}

The CMD is a helpful tool to confirm the reliability of our membership analysis and to provide some additional information about the HCMs for which we were not able to establish a secure membership based on their lithium content. Figure 7 shows the position of the eight UVES targets considered as Li members, plus the $11 \mathrm{HCMs}$ and the Giraffe members from Jeffries et al. (2014); we made the diagram using the photometry given by Jeffries et al. (2009) and also released to the GaiaESO consortium. For stars not included in this compilation, we have used the photometry from the Tycho-2 catalog (Høg et al. $2000)$. Both the distance modulus ( $\mathrm{DM}=7.76)$ and reddening $(E(B-V)=0.038)$ are taken from Jeffries et al. (2009). As expected, the majority of the stars fall in proximity or above the ZAMS in a sequence close to the $10 \mathrm{Myr}$ isochrone, although a few outliers are present. Among the UVES sample, there are six 
L. Spina et al.: Gaia-ESO Survey: the first abundance determination of the gamma Velorum cluster

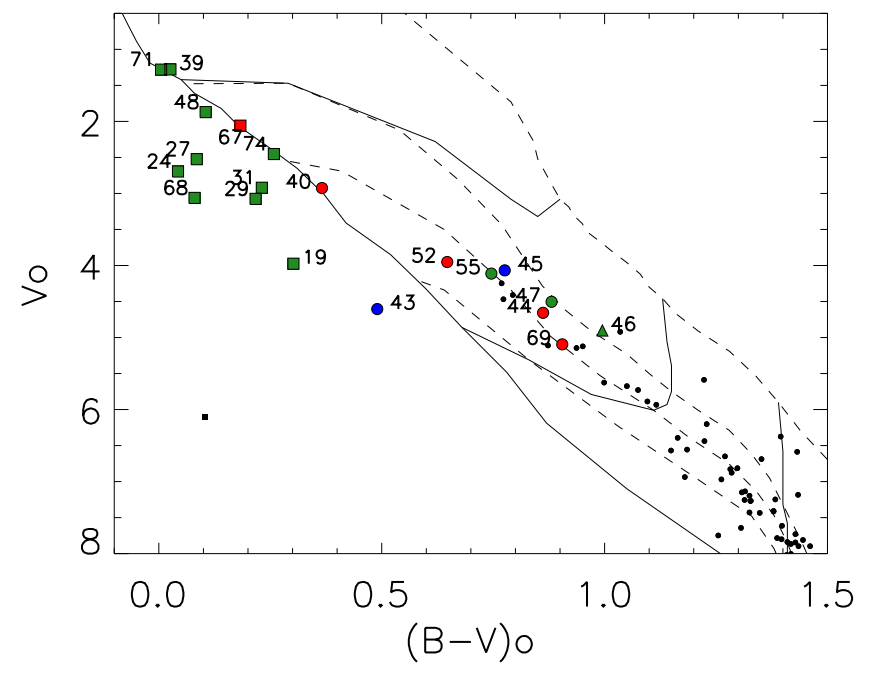

Fig. 7. $V_{0}$ vs. $(B-V)_{0}$ diagram of the UVES high-probability members (circles), HCM (squares) and Li-rich binary system (triangle). Stars are color coded according to the RV membership. Each star is labelled according to the ID number given in Table 1. The Giraffe members from Jeffries et al. (2014) are shown as black dots. The solid and dashed lines are the evolutionary tracks for $0.5,1$ and $2 M_{\odot}$ and isochrones for 1, 5, 10, 20 Myr and ZAMS from Siess et al. (2000) for a chemical composition with $Z=0.01$. The diagram is limited to the color-magnitude range relevant for the UVES targets.

HCMs (\#19, 24, 27, 68, 31, 29) and one Li member (\#43) lying significantly below the ZAMS, i.e., more than the $\pm 0.3 \mathrm{mag}$ spread in distance modulus found by Jeffries et al. (2009) (uncertainties on photometry and extinction are negligible for these stars). We will not consider these six HCMs for further analysis since they are likely field dwarfs. On the other hand, we note that star \#43 has an RV value slightly below $\left(\sim 1 \mathrm{~km} \mathrm{~s}^{-1}\right)$ our threshold for membership; however, its high EW(Li) and surface gravity $(\log g=4.03 \mathrm{dex})$ are consistent with those of other highprobability members. Star \#45, which lies slightly below the upper envelope of the Pleiades distribution in Fig. 6, is consistent with the other UVES Li members and the Giraffe members in the CMD. Thus, we include both these latter as likely members in the sample considered for the abundance analysis.

\subsection{Summary of the membership analysis}

In Table 2, we list the parameters of the 80 stars observed with UVES. In the last five columns, we give the membership status from the RV, surface gravity, $\mathrm{EW}(\mathrm{Li})$, and the position in the CM diagram: "M" stands for member, "N" for field contaminant, and "HCM" for hot candidate member. Table 5 summarizes the tally resulting from the analysis of the individual membership indicators. In total, there are eight high-probability members, as indicated by their lithium, plus one Li-rich SB2 that can be considered a likely member. Among the high-probability members, four are also RV members. There are also five HCMs that satisfy the criteria for membership based on $\log g$ and the CMD. In total, we have 14 likely members. Note that the membership of two out of the seven RV members is not confirmed by the lithium analysis, implying a contamination of about $30 \%$ in the RV sample.

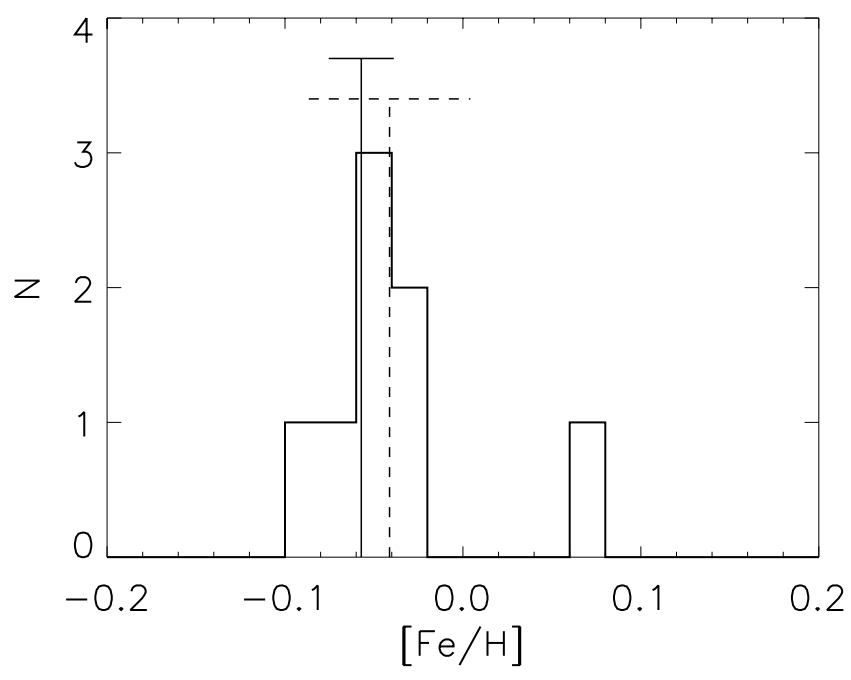

Fig. 8. Distribution of the iron abundance of the eight UVES highprobability cluster members. The mean values are $\langle[\mathrm{Fe} / \mathrm{H}]\rangle=-0.04 \pm$ $0.05 \mathrm{dex}$ (dashed line) and $\langle[\mathrm{Fe} / \mathrm{H}]\rangle=-0.057 \pm 0.018 \mathrm{dex}$ (solid line) discarding the star \#52 with $[\mathrm{Fe} / \mathrm{H}]=0.07$ dex.

\section{Abundance analysis of the members of gamma Velorum}

\subsection{Iron abundance}

Based on the eight UVES high-probability members, we obtain the $[\mathrm{Fe} / \mathrm{H}]$ distribution of gamma Velorum shown in Fig. 8. The mean iron abundance is $\langle[\mathrm{Fe} / \mathrm{H}]\rangle=-0.04 \pm 0.05 \mathrm{dex}$, where the error corresponds to $1 \sigma$ of the distribution. We recall that this $[\mathrm{Fe} / \mathrm{H}]$ value refers to a solar value of $\log n(\mathrm{Fe})=7.45$ (Grevesse et al. 2007). While seven of the eight stars have abundances in the narrow range -0.1 to -0.03 dex, the difference between the metallicity of J08095427-4721419 (\#52; $[\mathrm{Fe} / \mathrm{H}]=$ +0.07 dex) and the mean is larger than $\sim 2 \sigma$.

The membership of this star is based on the gravity, the presence of photospheric $\mathrm{Li}$, and on an RV consistent with that of the cluster. Other indirect supports come from the relatively high rotational velocity typical of young stars, a proper motion consistent with the other members of the cluster and high level of X-ray emission (Jeffries et al. 2009). Furthermore, the star exhibits an IR excess at $24 \mu \mathrm{m}$ that suggests the presence of a debris disk (Hernandez et al. 2008). The possible origin of the high-iron abundance of J08095427-4721419 is discussed in Sect. 5.3. If we excluded this member with a peculiar high metallicity, we would obtain a mean iron abundance of $-0.057 \pm 0.018$ dex. These results indicate that the members of gamma Velorum have a slightly subsolar iron abundance with a small dispersion. The mean iron abundance is compatible with that derived in other young open clusters of the solar neighborhood (Biazzo et al. 2011a), while the small scatter suggests a homogeneous iron abundance in gamma Velorum. Considering the two kinematic groups, we note that among the eight members only one star (J08110285-4724405, \#69) is more likely associated with Population A in Jeffries et al. (2014), while the remaining seven ones more likely belong to Population B. Hence, based on the UVES targets, we cannot make a comparative analysis of the abundances in terms of the two RV populations.

The much larger number of Giraffe members and the richer statistics allow for a more general study of the iron abundance distribution in gamma Velorum. In Fig. 9, we show the iron abundance of the Giraffe members from Jeffries et al. (2014) 


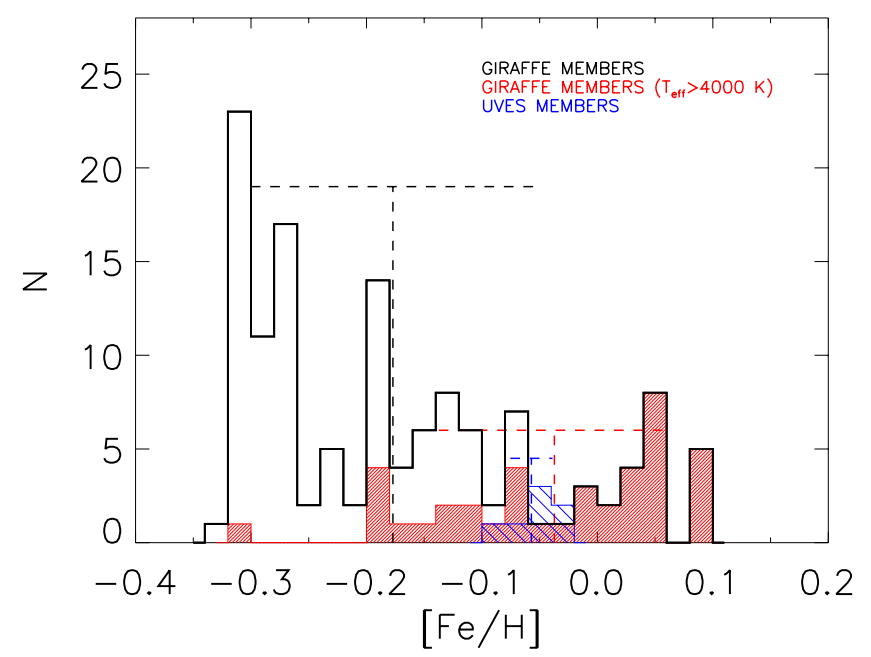

Fig. 9. Iron abundance of the Giraffe targets identified by Jeffries et al. (2014) as members (solid histogram). The other histograms show the iron abundance og the Giraffe members with $T_{\text {eff }}>4000 \mathrm{~K}$ (red) and that of the UVES members (blue).

with $v \sin i \leq 50 \mathrm{~km} \mathrm{~s}^{-1}$. The latter constraint is necessary because in the spectra of fast rotating stars the blending of the absorption features makes the iron abundance estimate highly uncertain. We find that $\langle[\mathrm{Fe} / \mathrm{H}]\rangle_{\text {Giraffe }}=-0.18 \pm 0.12$ dex is consistent within the errors, though somewhat lower, with the mean iron abundance based on the analysis of UVES spectra (Fig. 8). The different mean iron abundance and the broader width of the distribution (from -0.34 to +0.13 dex) are partly due to the lower resolution of the Giraffe spectra and also to the intrinsic difficulty of the analysis of cool stars. If we restrict the Giraffe sample to stars with $T_{\text {eff }}>4000 \mathrm{~K}$ ( $\sim 25 \%$ of the objects), we derive a mean value of $\langle[\mathrm{Fe} / \mathrm{H}]\rangle_{\text {Giraffe }}=-0.04 \pm 0.10 \mathrm{dex}$, with a greater similarity to that of the UVES sample. Note that a few stars with high metallicity are present in the Giraffe distribution, comparable to that of star J08095427-4721419. Whereas the larger typical uncertainties of the Giraffe determinations certainly contribute to broadening the $[\mathrm{Fe} / \mathrm{H}]$ distribution, we cannot exclude the possibility that the Giraffe sample also contains a number of as yet unidentified metal-rich outliers, as well as field contaminants or other difficult stars, for example binaries, which could affect the distribution.

Finally, considering the Giraffe members with $T_{\text {eff }}>$ $4000 \mathrm{~K}$, we plot in Fig. 10 their iron abundances as a function of the RVs. Although a group of stars with RV values ranging between 18.5 and $20 \mathrm{~km} \mathrm{~s}^{-1}$, likely belonging to Population B, have lower iron abundances with respect to the other stars, there is a significant scatter among the stars of the two populations without hints of abundance separations. Thus, we conclude that the two groups likely have the same iron abundance.

\subsection{Other elements}

The Gaia-ESO Survey has released for gamma Velorum the abundance of elements other than iron for stars with $v \sin i<20 \mathrm{~km} \mathrm{~s}^{-1}$. Unfortunately, this limits the analysis to two confirmed members only: J08095427-4721419 (\#52) and J08093304-4737066(\#45). The elemental abundances are listed in Table 6.

We see that for the cooler star (\#45) the abundances are within \pm 0.1 dex of the solar values, with the only exception

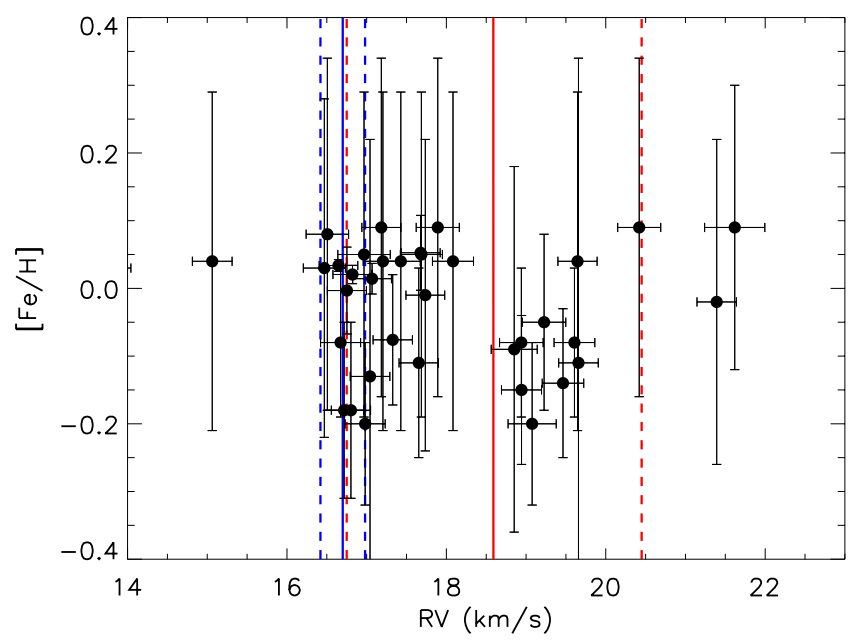

Fig. 10. Iron abundance of the Giraffe targets identified by Jeffries et al. (2014) as members and with $T_{\text {eff }}>4000 \mathrm{~K}$ as a function of their RVs. The red and blue solid lines mark the central RV of each young population identified by Jeffries et al. (2014). The dashed lines corresponds to $\pm 1 \sigma$ central value.

Table 6. Elemental abundances.

\begin{tabular}{lcc}
\hline \hline & $\begin{array}{c}\text { J08093304-4737066 } \\
\# 45\end{array}$ & $\begin{array}{c}\text { J08095427-4721419 } \\
\# 52\end{array}$ \\
\hline$T_{\text {eff }}^{\text {spec }}(\mathrm{K})$ & 5471 & 5756 \\
{$[\mathrm{Fe} / \mathrm{H}]$} & $-0.06 \pm 0.12$ & $+0.07 \pm 0.07$ \\
{$[\mathrm{Si} / \mathrm{Fe}]$} & $-0.10 \pm 0.08$ & $+0.18 \pm 0.20$ \\
{$[\mathrm{Mg} / \mathrm{Fe}]$} & $+0.02 \pm 0.14$ & $+0.30 \pm 0.40$ \\
{$[\langle\mathrm{Ti}\rangle / \mathrm{Fe}]$} & $-0.04 \pm 0.07$ & $+0.46 \pm 0.29$ \\
{$[\mathrm{Ca} / \mathrm{Fe}]$} & $+0.18 \pm 0.03$ & $+0.08 \pm 0.40$ \\
{$[\mathrm{Cr} / \mathrm{Fe}]$} & $+0.06 \pm 0.03$ & $+0.47 \pm 0.22$ \\
{$[\mathrm{Ni} / \mathrm{Fe}]$} & $-0.18 \pm 0.05$ & $+0.08 \pm 0.52$ \\
\hline
\end{tabular}

being calcium and nickel, which are enhanced and subsolar, respectively. The warmer star (\#52) shows abundances significantly larger than solar and very unusual for the solar neighborhood for most of the analyzed elements.

These abundances may, in principle, shed light on the reasons for the high metallicity found in star \#52 given that it is a metal-rich star; however, the errors are much larger than those obtained for \#45, with the exception of iron. Hence, we cannot attempt any conclusions based on the abundance ratios.

\subsection{The metal rich star J08095427-4721419}

J08095427-4721419, one of the eight high-probability members of gamma Velorum, has an iron abundance of $[\mathrm{Fe} / \mathrm{H}]=+0.07 \pm 0.07$ dex. We also notice that different analysis methods within the Gaia-ESO consortium have derived similar stellar parameters and enhanced metallicity (see Table 7). The position in the CMD reinforces the quality of the atmospheric parameters. Thus, we assume that this star is genuinely more metal-rich than other cluster members.

Based on statistical considerations, one would expect to detect one $2 \sigma$ outlier in a sample containing more than 20 stars; hence, the probability of having one outlier out of eight members is rather small, although not negligible. Under the assumption 
Table 7. Fundamental parameters of J08095427-4721419.

\begin{tabular}{lccc}
\hline \hline & $\begin{array}{c}T_{\text {eff }} \\
(\mathrm{K})\end{array}$ & $\begin{array}{c}\log g \\
(\mathrm{dex})\end{array}$ & $\begin{array}{c}{[\mathrm{Fe} / \mathrm{H}]} \\
(\mathrm{dex})\end{array}$ \\
\hline WG11 average & $5756 \pm 93$ & $4.25 \pm 0.16$ & $+0.07 \pm 0.07$ \\
WG12 average & $5864 \pm 112$ & $4.41 \pm 0.08$ & $+0.14 \pm 0.09$ \\
$\mathrm{WG} 12^{a}$ & $5944 \pm 57$ & $4.45 \pm 0.10$ & $+0.20 \pm 0.13$ \\
${\mathrm{WG} 12^{b}}^{5785 \pm 56}$ & $4.37 \pm 0.13$ & $+0.08 \pm 0.11$ \\
\hline
\end{tabular}

Notes. ${ }^{(a)}$ Iron EWs method. ${ }^{(b)}$ Comparison with a library of standard star spectra.

that the star is a genuine metal-rich cluster member, we propose the following scenario to explain it.

First, we believe that the chemical enrichment due to the explosion of a nearby $\mathrm{SN}$ is unlikely since it should have enriched the whole cloud and other members. A more likely process is the accretion of circumstellar rocky material onto the star that is mixed in the stellar convective envelope causing an overall metallicity enhancement (Laughlin \& Adams 1997). If the star is cool and young, its extended outer convection zone will effectively mix the accreted material with only a minimal metallicity enhancement. On the other hand, if the star is mostly radiative with a thin convective layer, the pollution could be much more important, leading to observable consequences.

Applying the scenario proposed by Laughlin \& Adams (1997), a solar-type star, like J08095427-4721419, starts its PMS contraction with a fully convective structure, but after $\sim 2 \mathrm{Myr}$ a radiative core appears that grows in mass as the star ages, shrinking the outer convective layers. Such a star maintains a thick convective envelope until about $10 \mathrm{Myr}$. For later-type stars, the growth of the radiative core takes more time and the final thickness of the convective layer is larger; in earlier-type stars, the radiative core develops quickly until a fully radiative configuration is reached. Circumstellar disks are found in most of young stellar objects and generally they accrete onto the central star during the first $10 \mathrm{Myr}$ when their internal structure is still mainly convective. The condensation of heavy elements could lead to the formation of rocky blocks or planets, however, which prevents a quick accretion of this material during the time when the star is mainly convective. In the last decade, several surveys have shown that a great number of extrasolar planets have surprisingly small orbits, suggesting that after their formation significant orbital migration takes place in the protoplanetary system. A possible outcome of this inward migration is that part of the planetary material reaches the central star even after the completion of the main accretion phases.

\subsection{A quantitative estimate of the effects of rocky material accretion on the mainly radiative PMS star J08095427-4721419}

Using $T_{\text {eff }}=5756 \mathrm{~K}$ and $L_{\mathrm{bol}}=2.5 L_{\odot}$ for J08095427-4721419 and the Siess models for a subsolar metallicity (Siess et al. 2000), we derive a stellar mass of $\sim 1.3 M_{\odot}$ and an age of 15-16 Myr. Thus, this star appears somewhat older than the average age of the cluster, but consistent with the age dispersion found by Jeffries et al. (2009, 2014). As we mentioned above, the Spitzer data show evidence for the presence of a debris disk (Hernandez et al. 2008). Thus, we can imagine that part of the circumstellar matter has condensed into hydrogendepleted rocks, or even planets, and that this rocky material has recently accreted onto the star. The Siess models also predict that such a star is almost fully radiative, but that about $5 \mathrm{Myr}$ in the past it had a thin convective layer of $\sim 0.05 M_{\odot}$. We now estimate the mass of heavy elements (expressed in Earth masses, $M_{\oplus} \sim 3 \times 10^{-6} M_{\odot}$ ) that must have been accreted onto the star during the last $5 \mathrm{Myr}$ to produce an iron enhancement similar to that observed in J08095427-4721419. For this purpose, we assume that the accreted material is mixed in a convective region containing $0.05 M_{\odot}$. We also assume that the star has an initial iron abundance equal to the average value of the other members $\left([\mathrm{Fe} / \mathrm{H}]_{\text {init }}=-0.057 \mathrm{dex}\right)$. Now, the rocky material, being hydrogen-depleted, has a mass ratio of metals $Z_{p}=1$, and we assume that such a rocky mass has the heavy element distribution as the solar mix given by Grevesse et al. (2007). Hence, a mixing of 50,60 , and $70 M_{\oplus}$ in the $0.05 M_{\odot}$ convective layer would be enough to produce a metallicity variation $(\Delta Z)$ of $3.0 \times 10^{-3}, 3.6 \times 10^{-3}$, and $4.1 \times 10^{-3}$, corresponding to a final iron abundance of $[\mathrm{Fe} / \mathrm{H}]_{\text {fin }}=+0.05,+0.07$ and +0.08 dex. This is just the right amount needed to explain the observed iron abundance. The same effect could be achieved by the accretion of two Jupiters $\left(M_{\mathrm{J}}=0.001 M_{\odot}\right)$ with a metallicity $Z=0.1$.

We recall that the mass of heavy elements currently contained in the planets of the solar system is estimated to be in the range 60-120 $M_{\oplus}$ (Wuchterl et al. 2000). This number is also in the range of heavy-element mass for exoplanets found by Miller \& Fortney (2011). Thus, our estimate of the accreted mass is consistent with these numbers. On the other hand, we have found only one star with a significant metallicity enhancement, whereas the presence of circumstellar disks is a frequent phenomenon around young stars and more than just one example should have been found in gamma Velorum. Our proposed scenario requires the refinement of several factors. First, the star must be of the right mass to possess a convective region that shrinks significantly while contracting. Then, this star should have the right amount of mass in the convective layer since otherwise the accreted metals could be too diluted or, conversely, enhanced with respect to the observed abundance. Third, the accretion episode must have occurred only after the star has had time to contract significantly for the retreat of the convection layer and this requires several Myr. The fact that J080954274721419 is a bit older than the other members of the cluster, judging from its isochronal age, supports our interpretation. A similar scenario has recently been suggested by Théado \& Vauclair (2012) in the context of the predicted modifications of the light element abundances of accreting exoplanet-host stars. Although the case of J08095427-4721419 is the only one found so far in gamma Velorum, we should also mention other examples of metal-rich stars in other solar or subsolar young clusters and SFRs (e.g., Wilden et al. 2002; Biazzo et al. 2011a).

\section{Conclusions}

In this paper, we have made use of the dataset provided by the Gaia-ESO Survey to identify the gamma Velorum members in the UVES sample and to study their elemental abundances, in particular to derive the mean cluster metallicity. The main findings can be summarized as follows:

i) The main result of this paper is the first metallicity estimation of the gamma Velorum cluster. We find that it has a slightly subsolar mean iron abundance: $\langle[\mathrm{Fe} / \mathrm{H}]\rangle=$ $-0.057 \pm 0.018 \mathrm{dex}$, if we exclude the metal-rich star J08095427-4721419. The analysis of other heavy element ( $\alpha$, iron peak, etc.) abundances for two members is not 
conclusive, given the very large uncertainties. This is the first estimate of the metallicity of gamma Velorum. When compared with the metallicity of other clusters belonging to the Vela complex and observed by the Gaia-ESO Survey (but whose analysis has not yet been completed), it will possibly allow us to put constraints on the star formation process in the complex.

ii) In order to determine the iron content of the cluster, we performed the membership analysis on the whole sample of targets. Using RVs, surface gravity and the presence of $\mathrm{Li}$ in the stellar atmospheres, we have identified eight highprobability members. We have also detected one SB2 system (J08093589-4718525, \#46) whose components both display a strong lithium line. This spectroscopic binary could be considered a likely member. Furthermore, we have identified five hot-candidate members of the cluster based on their position in the CMD.

iii) We have found a metal-rich member, J08095427-4721419. Its mass $\left(\sim 1.3 M_{\odot}\right)$ and age $(\sim 15 \mathrm{Myr})$ are consistent with an internal structure characterized by a thin convective envelope. We have suggested a scenario to account for the observed increase of the atmospheric abundances based on the accretion of $\sim 60 M_{\oplus}$ of rocky hydrogen-depleted material onto the star.

iv) The average metallicity derived from the Giraffe sample is similar to the average for UVES. A few metal-rich stars are also present in the Giraffe sample, but their presence may be due to the larger uncertainties and dispersion. Based on Giraffe sample, no major difference in the $[\mathrm{Fe} / \mathrm{H}]$ distribution is found for the two kinematic population identified by Jeffries et al. (2014).

On the more technical aspects, the comparison of the parameters from the UVES and Giraffe analysis of the same stars observed in gamma Velorum can be summarized as follows:

i) The Giraffe radial velocities of the first release are systematically higher with respect to the UVES values by $1.1 \pm$ $0.4 \mathrm{~km} \mathrm{~s}^{-1}$ (see Sacco et al. 2014 for a detailed discussion).

ii) The stellar parameters $\log g$ and $T_{\text {eff }}$ and the measured Li EWs are generally in good agreement, although small discrepancies are present for $T_{\text {eff }}>5500 \mathrm{~K}$ and $E W(\mathrm{Li})<30 \mathrm{~m} \AA$.

iii) There is a reasonable agreement between the iron abundances derived using Giraffe and UVES, but the intrinsic dispersion of the latter is significantly smaller.

Acknowledgements. We acknowledge the support from INAF and Ministero dell'Istruzione, dell'Università e della Ricerca (MIUR) in the form of the grant "Premiale VLT 2012". We also acknowledge the financial support from "Programme National de Cosmologie and Galaxies" (PNCG) of CNRS/INSU, France. The results presented here benefited from discussions in three GaiaESO workshops supported by the ESF (European Science Foundation) through the GREAT (Gaia Research for European Astronomy Training) Research Network Program (Science meetings 3855, 4127 and 4415). T.B. was funded by grant No. 621-2009-3911 from The Swedish Research Council. This research has made use of the SIMBAD database, operated at CDS, Strasbourg, France. E.J.A. acknowledges financial support from the "Ministerio de Economía y Competitividad" of Spain through grant AYA2010-17631. S.G.S, E.D.M., and V.Zh.A. acknowledge support from the Fundação para a Ciência e Tecnologia (Portugal) in the form of grants SFRH/BPD/47611/2008, SFRH/BPD/76606/2011, SFRH/BPD/70574/2010, respectively.

\section{References}

Adibekyan, V. Z., Delgado Mena, E., Sousa, S. G., et al. 2012a, A\&A, 547, A36 Adibekyan, V. Z., Santos, N. C., Sousa, S. G., et al. 2012b, A\&A, 543, A89

Biazzo, K., Randich, S., \& Palla, F. 2011a, A\&A, 525, A35

Biazzo, K., Randich, S., Palla, F., \& Briceño, C. 2011b, A\&A, 530, A19

Brandt, J. C., \& Maran, S. P. 1971, in BAAS, 3, 396

Cayrel, R. 1988, in The Impact of Very High S/N Spectroscopy on Stellar

Physics, eds. G. Cayrel de Strobel, \& M. Spite, IAU Symp., 132, 345

Cunha, K., \& Lambert, D. L. 1992, ApJ, 399, 586

Cunha, K., \& Lambert, D. L. 1994, ApJ, 426, 170

Cunha, K., Smith, V. V., \& Lambert, D. L. 1998, ApJ, 493, 195

D’Orazi, V., Randich, S., Flaccomio, E., et al. 2009, A\&A, 501, 973

Eldridge, J. J. 2009, MNRAS, 400, L20

Ercolano, B., \& Clarke, C. J. 2010, MNRAS, 402, 2735

Friel, E. D. 1995, ARA\&A, 33, 381

Gilli, G., Israelian, G., Ecuvillon, A., Santos, N. C., \& Mayor, M. 2006, A\&A, 449, 723

Gilmore, G., Randich, S., Asplund, M., et al. 2012, The Messenger, 147, 25

Gonzalez, G. 1998, in Brown Dwarfs and Extrasolar Planets, eds. R. Rebolo,

E. L. Martin, \& M. R. Zapatero Osorio, ASP Conf. Ser., 134, 431

Grevesse, N., Asplund, M., \& Sauval, A. J. 2007, Space Sci. Rev., 130, 105

Gustafsson, B., Edvardsson, B., Eriksson, K., et al. 2008, A\&A, 486, 951

Hernandez, J., Hartmann, L., Calvet, N., et al. 2008, ApJ, 686, 1195

Høg, E., Fabricius, C., Makarov, V. V., et al. 2000, A\&A, 355, L27

Humphreys, R. M. 1978, ApJS, 38, 309

James, D. J., Melo, C., Santos, N. C., \& Bouvier, J. 2006, A\&A, 446, 971

Jeffries, R. D., Naylor, T., Walter, F. M., Pozzo, M. P., \& Devey, C. R. 2009, MNRAS, 393, 538

Jeffries, R. D., Jackson, R. J., Cottaar, M., et al. 2014, A\&A, 563, A94

Johnson, J. A., Aller, K. M., Howard, A. W., \& Crepp, J. R. 2010, PASP, 122, 905

Jones, B. F., Shetrone, M., Fischer, D., \& Soderblom, D. R. 1996, AJ, 112, 186

Kang, W., Lee, S.-G., \& Kim, K.-M. 2011, ApJ, 736, 87

Laughlin, G., \& Adams, F. C. 1997, ApJ, 491, L51

Miller, N., \& Fortney, J. J. 2011, ApJ, 736, L29

Millour, F., Petrov, R. G., Chesneau, O., et al. 2007, A\&A, 464, 107

Mordasini, C., Alibert, Y., Benz, W., Klahr, H., \& Henning, T. 2012, A\&A, 541, A97

Neves, V., Santos, N. C., Sousa, S. G., Correia, A. C. M., \& Israelian, G. 2009, A\&A, 497, 563

North, J. R., Tuthill, P. G., Tango, W. J., \& Davis, J. 2007, MNRAS, 377, 415

Pasquini, L., Avila, G., Blecha, A., et al. 2002, The Messenger, 110, 1

Pettersson, B. 2008, Young Stars and Dust Clouds in Puppis and Vela, ed.

B. Reipurth, 43

Pozzo, M., Jeffries, R. D., Naylor, T., et al. 2000, MNRAS, 313, L23

Randich, S., \& Gilmore, G. 2013, Messenger, 154, 47

Sacco, G. G., Morbidelli, L., Franciosini, E., et al. 2014, A\&A, 565, A113

Santos, N. C., Israelian, G., \& Mayor, M. 2004, A\&A, 415, 1153

Santos, N. C., Melo, C., James, D. J., et al. 2008, A\&A, 480, 889

Schaerer, D., Schmutz, W., \& Grenon, M. 1997, ApJ, 484, L153

Sherry, W. H., Walter, F. M., Wolk, S. J., \& Adams, N. R. 2008, AJ, 135, 1616

Sicilia-Aguilar, A., Hartmann, L., Calvet, N., et al. 2006, ApJ, 638, 897

Siess, L., Dufour, E., \& Forestini, M. 2000, A\&A, 358, 593

Slawson, R. W., \& Reed, B. C. 1988, JRASC, 82, 276

Smiljanic, R., Korn, A., Bergemann, M., et al. 2014, A\&A, submitted

Smith, L. F. 1968, MNRAS, 141, 317

Soderblom, D. R., Jones, B. F., Balachandran, S., et al. 1993, AJ, 106, 1059

Spezzi, L., de Marchi, G., Panagia, N., Sicilia-Aguilar, A., \& Ercolano, B. 2012, MNRAS, 421, 78

Stauffer, J. R., Schultz, G., \& Kirkpatrick, J. D. 1998, ApJ, 499, L199

Sushch, I., Hnatyk, B., \& Neronov, A. 2011, A\&A, 525, A154

Théado, S., \& Vauclair, S. 2012, ApJ, 744, 123

van Leeuwen, F. 2007, A\&A, 474, 653

Wilden, B. S., Jones, B. F., Lin, D. N. C., \& Soderblom, D. R. 2002, AJ, 124, 2799

Wuchterl, G., Guillot, T., \& Lissauer, J. J. 2000, Protostars and Planets IV (Tucson: University of Arizona press), 1081

Yasui, C., Kobayashi, N., Tokunaga, A. T., Saito, M., \& Tokoku, C. 2010, ApJ, 723, L113 